Original

\title{
Ensayo de campo de un procedimiento de investigación de casos de enfermedades de origen laboral
}

\section{Testing a procedure to investigate occupational related diseases}

\author{
José María Roel Valdés ', Jerónimo Maqueda Blasco \\ 1. INVASSAT (Instituto Valenciano de Seguridad y Salud en el Trabajo), Alicante \\ 2. Escuela Nacional de Medicina del Trabajo, Instituto de Salud Carlos III, Madrid. \\ Recibido: 26-03-13 \\ Aceptado: 20-05-13
}

\section{Correspondencia}

José María Roel Valdés

Jefe de Servicio Especialidades Preventivas

Centro Territorial de Seguridad y Salud

INVASSAT-ALICANTE

Tfno: 965934939

roel_jos@gva.es

Resumen

Los datos de la Encuesta Nacional de Gestión de la Seguridad y Salud en las Empresas pone de manifiesto una escasa práctica en la investigación de casos de enfermedades profesionales o relacionadas con el trabajo. En este contexto se desarrolla este proyecto dirigido al desarrollo y ensayo de un procedimiento de investigación de casos de enfermedades profesionales o relacionadas con el trabajo, que permita orientar, armonizar y sistematizar la recogida de información en el transcurso de la investigación de este tipo de casos. En una primera fase un grupo investigadores del Instituto Valenciano de Seguridad y Salud en el Trabajo y de la Escuela Nacional de Medicina del Trabajo del Instituto de Salud Carlos III desarrollaron un procedimiento de investigación de casos de enfermedades laborales (profesionales o relacionadas con el trabajo), que se sometió mediante un taller al consenso con un grupo de expertos procedentes de distintos grupos de interés: Sindicatos, Servicios de Prevención Propios y Ajenos, Mutuas, Inspección de Trabajo, órganos técnicos de las CCAA, Sociedades de Medicina del Trabajo y Organismos Públicos de Investigación y Universidad. El procedimiento resultante se ensayó en 21 casos incidentes de enfermedades laborales, se evaluaron un total de 26 indicadores en tres dimensiones: necesidad, aplicabilidad y valoración general, medidos por una escala tipo Likert, evaluando el grado de acuerdo entre aplicadores mediante el procedimiento de estandarización del Instrumento AGREE. Los resultados ponen de manifiesto en 19 indicadores un grado de consenso superior al $80 \%$ y en 10 un grado de consenso superior al $90 \%$, no existiendo disenso en ningunos de los indicadores analizados, lo que permite concluir que el procedimiento ensayado cubre las necesidades de información en la investigación de casos de enfermedades laborales.

Med Segur Trab (Internet) 2013; 59 (231) 227-234

Palabras Clave: Enfermedades profesionales, investigación, procedimiento

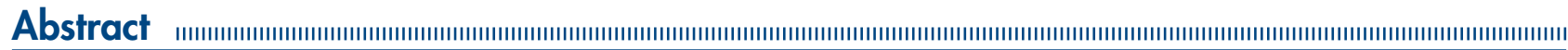

Data from the National Survey of Safety and Health Management at Companies evidence gaps the investigation of cases of occupational diseases. In this context this project aims to developed and test an investigation procedure of occupational diseases, in order to guide, harmonize and systematize the collection of information in the course of the investigation of this cases.
\end{abstract}


In the first phase a group of researchers from the Safety al Occupational Health of Valencia the National School of Occupational Medicine developed a procedure for investigate cases of occupational diseases that was submitted to a consensus workshop with a group of experts from various key agents: Employees Organizations, Prevention Services, Mutuas, Labour Inspectorate, Technical Institutes from the Autonomous Communities, Societies of Occupational Medicine, Public Research Institutes and University.

The resulting procedure was tested on 21 incident cases of occupational diseases, we evaluated a total of 26 indicators in three dimensions: needs, relevance and overall opinion, as measured by a Likert scale, assessing the degree of agreement between applicators by the standardization procedure of the AGREE evaluation method.

The results show on 19 indicators a level of consensus upper than $80 \%$ and for others 10 indicators a level of consensus upper than $90 \%$, there being no dissent in any of the indicators analyzed. These results leading to the conclusion that the tested procedure covers the information needs in the investigation of cases of occupational diseases.

Med Segur Trab (Internet) 2013; 59 (231) 227-234

Key words: Occupational diseases, investigation, procedure. 


\section{INTRODUCCIÓN}

Los estudios de investigación sobre enfermedades laborales vienen evidenciando que la mortalidad por enfermedades laborales es superior a la mortalidad por accidentes ${ }^{1}$.

En España, las estimaciones existentes señalan que unos 15.300 trabajadores mueren cada año a causa de enfermedades laborales ${ }^{2}$. Si consideramos que la mortalidad por accidente de trabajo se ha venido manteniendo en alrededor de 1000 trabajadores por año ${ }^{3}$, la mortalidad por enfermedades laborales superaría en unas 15 veces a la de accidentes.

Así mismo, en los últimos años se viene incidiendo sobre la importancia de los costes económicos que supone la falta de políticas preventivas de las enfermedades profesionales.

En el estudio de costes sanitarios de cáncer de pulmón y cáncer de vejiga de origen laboral se ha establecido una estimación de alrededor de 88 millones de euros, considerándola una estimación conservadora dado la limitación del acceso a determinado tipo de datos ${ }^{4}$.

Por otro lado, nuestro marco normativo de prevención de riesgos laborales obliga a la investigación de los daños para la salud y a la revisión de la evaluación de riesgos y planificación preventiva en función de los resultados de la misma ${ }^{5,6,7,8}$.

De forma progresiva, se han venido desarrollando diversos protocolos que abordan la investigación de enfermedades laborales desde el punto de vista epidemiológico, como el de Comunidad de Murcia ${ }^{9}$ Uno de los protocolos epidemiológicos, que llevan en funcionamiento desde hace más tiempo, es la Red Centinela de Navarra .Incorpora a los Médicos de Atención Primaria a la detección y comunicación de una serie de enfermedades centinela $^{10}$.

Tiene particular interés el Sistema de Información Sanitaria y Vigilancia Epidemiológica Laboral (SISVEL) ${ }^{11}$, modelo implantado en la Comunidad Valenciana. Establece la comunicación de enfermedades laborales desde los Médicos de Atención Primaria de la Comunidad Valenciana y los Servicios de Prevención de Riesgos Laborales a través de la red informática de la Agencia de Salud. Abarca a las enfermedades más frecuentes y sus resultados han ido consolidándose en los últimos años. La implantación gradual de este sistema, actualmente presente en el cien por cien de los centros de atención primaria y el 70 por ciento de los centros de especializada, ha permitido registrar en 2012 más de 1.300 casos de sospecha de enfermedad profesional.

Sin embargo, sigue existiendo una gran desproporción en los medios y recursos que se dedican a la investigación de los casos de accidentes de trabajo y los que se aplican para la investigación de los casos de enfermedades laborales.

De acuerdo a los resultados de la Encuesta Nacional de Gestión de Seguridad y Salud en las empresas ${ }^{12}$ casi el $30 \%$ de los casos de enfermedades profesionales no se investigan, frente a un $16 \%$ de los accidentes de trabajo.

Para la investigación directa de los casos de accidente existen diferentes modelos y métodos y es una práctica que está claramente asumida dentro de la cultura de la prevención de riesgos laborales. Por el contrario, sigue siendo una asignatura pendiente dentro la actividad preventiva en los lugares de trabajo, enfocar las enfermedades laborales cómo daños que se deban investigar directa e individualmente.

Por todo ello, y dada la transcendencia que tienen las enfermedades laborales tanto en mortalidad, como en costes económicos y sociales, el objetivo de este estudio es el de desarrollar un procedimiento armonizado en la investigación de casos de enfermedad profesional o relacionada con el trabajo que sirva como herramienta de aplicación práctica en la identificación de las sus causas.

Este objetivo se alinea con las necesidades actualmente existentes de profundizar en el conocimiento de las enfermedades laborales dentro del ámbito de la Prevención de Riesgos Laborales. 


\section{MATERIAL Y MÉTODOS}

El ensayo se realizó sobre casos incidentes de enfermedades con sospecha de relación laboral, ocurridos entre octubre de 2011 y enero de 2012, en el ensayo participaron un total de 13 expertos pertenecientes a 5 instituciones proveedoras de servicios de prevención a la empresa.

Se seleccionaron todos los casos ocurridos en ese periodo de tiempo e investigados por las entidades participantes en el ensayo.

Cada investigador, cumplimentó una encuesta de evaluación tras finalizar la investigación de cada uno de los casos.

La encuesta de evaluación comprendía un total de 26 indicadores correspondientes a las dimensiones de: Necesidad de la Información, es decir, si la información que se registra en el procedimiento es necesaria para la resolución del caso. Suficiencia de la Información, es decir si la información registrada en el procedimiento era considerada como suficiente para poder extraer conclusiones efectivas y Valoración General de su utilidad en el caso investigado, es decir, si el procedimiento resultó útil para extraer conclusiones e identificar medidas preventivas (tabla I).

Tabla I. Número de indicadores por dimensión evaluada del procedimiento, tras la investigación del caso

\begin{tabular}{lc}
\hline & $\mathbf{N}^{\circ}$ Indicadores \\
\hline Valorar si la información que se registra en el apartado correspondiente "ES NECESARIA & 10 \\
PARA DOCUMENTAR LA INVESTIGACIÓN DEL CASO". & \\
Valorar silas notas aclaratorias a los puntos respectivos, "FACILITAN LACUMPLIMENTACIÓN & 10 \\
DEL APARTADO CORRESPONDIENTE". & 6 \\
Valoración general de la aplicación del procedimiento en el caso investigado. & 6 \\
\hline
\end{tabular}

El acuerdo entre los investigadores con cada uno de los indicadores valorados se recogió en una escala tipo Likert del 1 al 7 correspondiendo el valor 1 con el menor nivel de acuerdo y el valor 7 con el máximo nivel de acuerdo.

Para cada uno de los indicadores se realizó un análisis descriptivo de la media y moda y un análisis del nivel de acuerdo entre la totalidad de casos investigados.

La estimación del grado de acuerdo se determinó mediante el método de estandarización del Instrumento AGREE ${ }^{\odot}$.

La puntuación estandariza obtenida se agrupó en seis grados de consenso (tabla II).

Tabla II. Grados de consenso

\begin{tabular}{lc}
\hline \multicolumn{1}{c}{ Grado } & Puntuación estandarizada \\
\hline Excelente & $100-90$ \\
Alto & $89-70$ \\
Medio & $69-50$ \\
Bajo & $49-30$ \\
Muy Bajo & $29-10$ \\
Disenso & $9-0$ \\
\hline
\end{tabular}

Los investigadores recibieron el procedimiento consolidado, la encuesta de evaluación $\mathrm{y}$ un documento instructivo del trabajo de campo. 


\section{RESULTADOS}

En total se investigaron 21 enfermedades, de las cuales 7 correspondían a procesos músculo esqueléticos, 4 a enfermedades por agentes biológicos, 3 a enfermedades por exposición a amianto, 2 a hipoacusias por ruido 5 a diferentes diagnósticos (tabla III).

Tabla III. Diagnósticos de los casos investigados

\begin{tabular}{lc}
\hline \multicolumn{1}{c}{ Diagnóstico } & n \\
\hline Bursitis & 1 \\
Conjuntivitis & 2 \\
Epicondilitis & 2 \\
Hipoacusia & 2 \\
Neumoconiosis por asbesto & 1 \\
Placas pleurales & 1 \\
Enf. Respiratoria (sin especificar) & 1 \\
Asma & 1 \\
Neumoconiosis & 2 \\
Sarampión & 1 \\
STC & 4 \\
Sensibilizaciónespiramicina & 1 \\
TBC & 1 \\
No comunicado el diagnóstico & 1 \\
Total & 21 \\
\hline
\end{tabular}

En relación al origen de los casos investigados en el $64 \%$ de los casos provenía de partes de enfermedad profesional declarados y en un $36 \%$ eran investigaciones por sospecha de enfermedad laboral.

La puntuación estandarizada obtenida al evaluar, por los investigadores, si la información recogida en el procedimiento era "necesaria" para documentar la investigación del caso investigado fue superior al 90\% (Grado de consenso excelente) en 6 de los 10 apartados (tabla IV).

Tabla IV. Grado de consenso para los casos investigados y puntuación estandarizada por apartado del procedimiento

"ES NECESARIA PARA DOCUMENTAR LA INVESTIGACIÓN DEL CASO"

\begin{tabular}{llc}
\hline $\begin{array}{c}\text { Grado de } \\
\text { consenso }\end{array}$ & \multicolumn{1}{c}{ Apartado del procedimiento } & $\begin{array}{c}\text { Puntuación } \\
\text { estandarizada \% }\end{array}$ \\
\hline \multirow{2}{*}{ EXCELENTE } & Descripción del proceso actual de la enfermedad & 100,0 \\
& Conclusiones médicos laborales & 100,0 \\
& Historia laboral & 96,0 \\
& Conclusiones de la investigación & 92,1 \\
& Descripción de las condiciones de trabajo y de riesgo para la enfermedad investigada & 90,5 \\
& Medidas preventivas y/o de protección a introducir en el puesto de trabajo & 90,5 \\
\hline \multirow{2}{*}{ ALTO } & Descripción de la actividad de la empresa & 87,3 \\
& Gestión preventiva & 81,0 \\
\hline \multirow{2}{*}{ MEDIO } & Equipos de protección individual & 75,4 \\
\hline
\end{tabular}

Con respecto a la evaluación del grado acuerdo entre los investigadores sobre la facilidad que suponían las notas aclaratorias para la cumplimentación de los diferentes apartados del procedimiento (tabla V), se obtuvo una puntuación estandarizada superior 
al 90\% (Grado de consenso excelente) en cuatro de los 10 apartados y en los otros seis el grado de consenso obtuvo valores ente el $70 \%$ y $90 \%$ (Grado de consenso alto).

Tabla V. Grado de consenso para los casos investigados y puntuación estandarizada para las notas aclaratorias a los apartados del procedimiento

"FACILITAN LA CUMPLIMENTACIÓN DEL APARTADO CORRESPONDIENTE"

\begin{tabular}{llc}
\hline $\begin{array}{c}\text { Grado de } \\
\text { consenso }\end{array}$ & \multicolumn{1}{c}{ Apartado del procedimiento } & $\begin{array}{c}\text { Puntuación } \\
\text { estandarizada \% }\end{array}$ \\
\hline \multirow{4}{*}{ EXCELENTE } & Historia laboral & 96,0 \\
& Descripción del proceso actual de la enfermedad & 94,4 \\
& Conclusiones médicos laborales & 93,7 \\
& Equipos de protección individual & 93,7 \\
\hline \multirow{3}{*}{ ALTO } & Descripción de las condiciones de trabajo y de riesgo para la enfermedad investigada & 87,3 \\
& Medidas de protección colectiva & 84,1 \\
& Conclusiones de la investigación & 81,7 \\
& Medidas preventivas y/o de protección a introducir en el puesto de trabajo & 78,6 \\
& Descripción de la actividad de la empresa & 78,6 \\
& Gestión preventiva & 77,0 \\
\hline
\end{tabular}

En relación con el grado de consenso de los investigadores sobre la operatividad de la información recogida durante la aplicación del procedimiento en lo que se refiere a facilitar el establecimiento de conclusiones sobre la naturaleza de la enfermedad y en la identificación de medidas preventivas a introducir en la empresa (tabla VI), se obtuvo una puntuación estandarizada superior al 70\% (Grado de consenso alto), siendo superior en el aspecto que se refería al establecimiento de conclusiones sobre la naturaleza profesional o no de la enfermedad.

Tabla VI. Grado de consenso para los casos investigados y puntuación estandarizada sobre la información recogida durante la aplicación del procedimiento

\begin{tabular}{llc}
\hline $\begin{array}{c}\text { Grado de } \\
\text { consenso }\end{array}$ & & $\begin{array}{c}\text { Puntuación } \\
\text { estandarizada \% }\end{array}$ \\
\hline \multirow{3}{*}{ ALTO } & $\begin{array}{l}\text { Le han facilitado establecer conclusiones respecto a la naturaleza de la enfermedad } \\
\text { (profesional, relacionada con el trabajo o común) del caso investigado. }\end{array}$ & 81,0 \\
& $\begin{array}{l}\text { Le han facilitado, en el caso investigado, la identificación de medidas preventivas } \\
\text { a introducir en la empresa. }\end{array}$ & 70,6 \\
\hline
\end{tabular}

Por último el análisis del grado de consenso de los investigadores sobre la valoración global del procedimiento en el caso investigado (tabla VII), todos los ítems presentan valores superiores al 70\% (Grado de consenso alto), siendo superior en el aspecto que se refiera al procedimiento como guía de investigación y como instrumento de sistematización en la recogida de la información.

Tabla VII. Grado de consenso para los casos investigados y puntuación estandarizada para la aplicación del procedimiento en el caso investigado

\begin{tabular}{llc}
\hline $\begin{array}{c}\text { Grado de } \\
\text { consenso }\end{array}$ & & $\begin{array}{c}\text { Puntuación } \\
\text { estandarizada \% }\end{array}$ \\
\hline \multirow{2}{*}{ ALTO } & Le ha permitido sistematizar la recogida de información & 82,5 \\
& Le ha guiado en el proceso de investigación & 81,7 \\
& La ha permitido identificar la información que debe trasladar a cada uno de los & 80,2 \\
& agentes y personas implicadas (trabajador, empresario, servicio de prevención, etc.) & \\
& Los algoritmos le han facilitado pautar el proceso de investigación & 77,0 \\
\hline
\end{tabular}




\section{CONCLUSIONES}

El procedimiento de Investigación de Casos de Enfermedades Laborales que se propone se ha diseñado recogiendo la opinión de los expertos que representan los distintos ámbitos potencialmente usuarios del mismo reflejando los puntos de vista tanto de trabajadores como de instituciones reguladoras y proveedores de servicios de Salud Laboral.

Si bien el número de enfermedades investigadas ha sido limitado a 21 casos, la variabilidad en el número de investigadores de campo implicados así como la diversidad de los casos estudiados, hablan a favor de la reproducibilidad de los resultados en la aplicación del procedimiento a sucesos distintos obteniendo resultados similares.

Los resultados del ensayo de campo realizado en la investigación de casos incidentes de enfermedades laborales, entendiendo éstas como enfermedades profesionales o relacionadas con el trabajo ponen de manifiesto, la existencia de un alto nivel de acuerdo en las respuestas del grupo de expertos que han aplicado el procedimiento.

En la mayoría de los ítems, el grado de consenso obtenido ha sido de Excelente o Alto en las dimensiones relativas a: Necesidad, Suficiencia y Utilidad del procedimiento aplicado.

Desde el punto de vista de la aplicabilidad el procedimiento ha permitido sistematizar y guiar el proceso de investigación. En lo que se refiere a su utilidad el procedimiento ha resultado útil para identificar el origen laboral de la enfermedad investigada, hecho que resulta relevante desde el punto de vista de mejorar de la notificación de las enfermedades profesionales, así como ha permitido identificar la medidas preventivas necesarias.

Estos resultados nos permiten concluir que el pprocedimiento de Investigación de Enfermedades Laborales sometido a ensayo es recomendable para su aplicación en campo para el estudio e investigación de casos de enfermedades laborales.

Su progresiva adaptación al progreso en materia de los cambios que pueda surgir en el ámbito de las enfermedades laborales, hace necesario que en el futuro de realicen nuevas evaluaciones que permitan estudiar y perfeccionar su aplicabilidad con el conocimiento adquirido a través de su utilización.

\section{AGRADECIMIENTOS}

María Ascensión Maestre Naranjo (Hospital Puerta de Hierro. Comunidad de Madrid); Reyes de la Cerda Sanchez (Sociedad de Prevención Fraternidad Muprespa); Vicente Ramón Reig Pastor (Sociedad de Prevención Fraternidad Muprespa); Antonio Víctor Sanz Smith (Sociedad de Prevención Fraternidad Muprespa); Jose Miguel Rodriguez Lopez (Sociedad de Prevención Fraternidad Muprespa); Leopoldo Alvarez Martín (Sociedad de Prevención Fraternidad Muprespa); $M^{a}$ Teresa Peralta García (Unimat Prevención); $M^{a}$ Jose Martínez Garcia (Unimat Prevención); Cristina Silva Gomez. (Unimat Prevención); Inés Borja Navarro (Unimat Prevención); Juan Navarro Bosch (Unimat Prevención); Elisabeth Purti Pujals(Sociedad de Prevención de Asepeyo); Carmen Molina (OTP Servicio de Prevención); Jordi Delclós Clanchet (Universitat Pompeu Fabra); Manuel Fernández Braso (UGT); José Manuel Gómez (ASPREM); Adrián González Martín (Ministerio de Trabajo e Inmigración); Teresa González (IRSST- Comunidad de Madrid); Francisco de la Gala Sánchez (SEMST); Loly Fernández Carou (CCOO); Carmen Mancheño (CCOO); María José López Jacob (ISTAS); Miguel Ángel Zamarrón (UGT); Javier Maestro Acosta (Ministerio de Trabajo e Inmigración); Manuel Martínez Vidal (Comunidad de Madrid); Clara Guillén Subirán (AEEMT); Claudia Naroki Flaminman (ISTAS); María Menéndez (CCOO); Coral Sainz Pinós (Hospital Puerta de Hierro, Comunidad de Madrid). 


\section{REFERENCIAS BIBLIOGRÁFICAS}

1. Païvï Hamalaïnen, Jukka Takala and Kaija Leena Saarela. Global Estimates of Fatal Work-Related Diseases; American Journal of Industrial Medicine. 2007; 50: 28-4.

2. García AM, Gadea R, López V. Estimación de la mortalidad atribuible a enfermedades laborales en España 2004. Rev Esp Salud Pública. 2007; 81: 261-270.

3. Ministerio de Empleo y Seguridad Social. Anuario de Estadísticas Laborales y de Asuntos Sociales. [monografía en internet]. Madrid: Ministerio de Empleo y Seguridad Social; 2011 [acceso 6 de mayo de 2013]. Disponible en: http://www.empleo.gob.es/estadisticas/ANUARIO2011/welcome.htm.

4. Montserrat García Gómez, Rosa Urbanos Garrido, Rosario Castañeda López y Patricia López Menduiña. Costes sanitarios directos de las neoplasias de pulmón y vejiga de origen laboral en España en 2008. Rev Esp Salud Pública. 2012; 86: 127-138.

5. Ley de Prevención de Riesgos Laborales. Ley 31/1995 de 8 de noviembre. Boletín Oficial del Estado, $\mathrm{n}^{\circ} 269,(10-11-1995)$

6. ORDEN TIN/2504, de 20 de septiembre de 2010, por la que se desarrolla el Real Decreto 39/1997, de 17 de enero, por el que se aprueba el Reglamento de los Servicios de Prevención, en lo referido a la acreditación de entidades especializadas como servicios de prevención, memoria de actividades preventivas y autorización para realizar la actividad de auditoría del sistema de prevención de las empresas. Boletín Oficial del Estado, $n^{\circ} 235$, (28 de septiembre de 2010)

7. Real Decreto $843 / 2011$, de 17 de junio, por el que se establecen los criterios básicos sobre la organización de recursos para desarrollar la actividad sanitaria de los servicios de prevención. Boletín Oficial del Estado, $\mathrm{n}^{\circ} 158$ (4 de julio de 2011).

8. Ley Reguladora de la Juridisción Social. Ley 36/2011, de 10 de octubre. Boletín Oficial del Estado, n ${ }^{\circ} 245$, (11 de Octubre de 2011).

9. Instituto de Seguridad y Salud Laboral [sede Web]. Murcia: Región de Murcia; [acceso 6 de mayo de 2013]. Protocolo de Investigación de Enfermedades Profesionales. Disponible en: http://www.carm.es/web/pag ina?IDCONTENIDO $=3508 \&$ RASTRO $=\mathrm{c} 722 \$ \mathrm{~m} \& I D T I P O=100$

10. V. García López. Evaluación el programa de Vigilancia Epidemiológica en Salud Laboral: Red de Médicos Centinela de Salud Laboral en Navarra, (1998-2007). An Sist Sanit Navar. 2011; 34 (3): 419-30.

11. Consellería de Sanidad [sede Web]. Valencia: Genralitat Valenciana. [acceso 23 de abril de 2013]. Guía para la comunicación de sospechas de enfermedades profesionales. Disponible en: http://www.sp.san. gva.es/DgspPortal/docs/guia_com_enf_sos.pdf.

12. Antonia Almodovar Molina, Francisco Javier Pinilla García. Encuesta Nacional de la Gestión de la Salud y Seguridad en las Empresas (ENGE 2009). [monografía en internet]. Madrid: Instituto Nacional de Seguridad e Higiene en el Trabajo; 2009 [acceso 6 de mayo de 2013]. Disponible en: http://www.oect.es/ Observatorio/Contenidos/InformesPropios/Desarrollados/Ficheros/Informe_\%20ENGE\%202009.pdf. 